\title{
Characterization of a cDNA from Beta maritima that confers nickel tolerance in yeast
}

\author{
Gonensin O. Bozdag a , Alaattin Kaya ${ }^{\mathrm{a}}$, Ahmet Koc ${ }^{\mathrm{a}}$, Gundula A. Noll ${ }^{\mathrm{b}}$, Dirk Prüfer ${ }^{\mathrm{b}}$, Hüseyin Caglar Karakaya ${ }^{\mathrm{a}, *}$ \\ a Izmir Institute of Technology, Department of Molecular Biology and Genetics, 35430 Urla, Izmir, Turkey \\ ${ }^{\mathrm{b}}$ Westphalian Wilhelms-University Münster, Institute for Biochemistry and Biotechnology of Plants, D-48143 Münster, Germany
}

\section{A R T I C L E I N F O}

Article history:

Accepted 21 January 2014

Available online 31 January 2014

\section{Keywords:}

Nickel

Beta maritima

Yeast

Transporter protein

Ion homeostasis

Heavy metal toxicity

\begin{abstract}
A B S T R A C T
Nickel is an essential micronutrient due to its involvement in many enzymatic reactions as a cofactor. However, excess of this element is toxic to biological systems. Here, we constructed a cDNA library from Beta maritima and screened it in the yeast system to identify genes that confer resistance to toxic levels of nickel. A cDNA clone (NIC6), which encodes for a putative membrane protein with unknown function, was found to help yeast cells to tolerate toxic levels of nickel. A GFP fused form of Nic6 protein was localized to multivesicular structures in tobacco epidermal cells. Thus, our results suggest a possible role of Nic6 in nickel and intracellular ion homeostasis.
\end{abstract} (c) 2014 Elsevier B.V. All rights reserved.

\section{Introduction}

At the end of the 1970's there was enough evidence showing that nickel is an essential element for diverse organisms including bacteria and animals (Eskew et al., 1984). For higher plants, such correlation was only confirmed at the end of the 1980s, and since then nickel has been considered as an essential element for higher plant growth (Eskew et al., 1984).

Nickel, mainly found in soil as in ionic form $\left(\mathrm{Ni}^{2+}\right)$ (Sajwan et al., 1996), enters to the plant cells mostly via root tissue, and then transported to the various plant organs (Sajwan et al., 1996). The uptake of nickel from soil is driven by active and passive transport, but the balance between these two systems was determined depending on to the soil nickel and pH levels (Peralta-Videa et al., 2002; Seregin and Kozhevnikova, 2006). High pH levels ( $\mathrm{pH}>8$ ) and existence of different metal ions in soil were reported to have an inhibitory effect on nickel uptake (Yusuf et al., 2011), and this might select for active transport over passive diffusion. Even though main rout of nickel translocation in plants is from roots to leaves, direct entry of nickel through the leaf tissue is an alternative and highly dynamic strategy Sajwan and colleagues showed that, $37 \%$ of the nickel introduced via leaf tissue, is transported into different tissues (Sajwan et al., 1996).

The primary role of nickel is its ability to bind to some specific enzymes as a co-factor, and subsequently bring them to functionally active

Abbreviations: CDF, cation diffusion facilitator; CASP, casparian strips; MTP, metal tolerance protein; GFP, green fluorescence protein; ICP-MS, inductively coupled plasma mass spectrometry; CLSM, confocal laser scanning microscopy.

* Corresponding author. Tel.: +90 232 7507527; fax: +90232 7507509.

E-mail address: caglarkarakaya@iyte.edu.tr (H.C. Karakaya). state (Watt and Ludden, 1999). The first identified nickel enzyme was Jack Bean urease which requires nickel as an essential element for its activity (Dixon et al., 1975). Urease converts urea to ammonia and carbamate by using water and while doing this it requires nickel in its active site. When plants are grown in soils containing urea as a nitrogen source, nickel becomes essential for the integrity of these plants; without nickel supply, urease cannot form its functionally active state, and this leads to the accumulation of urea as a highly toxic component (Eskew et al., 1984). Beside urease, enzymes such as hydrogenases, methyl coenzyme $\mathrm{M}$ reductase, $\mathrm{CO}$ dehydrogenase are defined as nickel enzymes with nickel biding sites, and all of these enzymes have bacterial origins (Walsh and Ormejohnson, 1987).

Until now, studies about nickel toxicity in plants have mostly focused on nickel hyperaccumulators that are capable of accumulating extremely high levels of nickel in their cell compartments. In nature, about 300 plant species have been identified as nickel hyperaccumulators (Boominathan and Doran, 2002). The strategies to produce plants that are resistant to toxic levels of nickel mainly depend on understanding the genetic and biochemical features of these hyperaccumulators.

Organisms have conserved mechanisms to detoxify heavy metals. Toxic metals can be pumped out of cells, their influx can be slowed down or they can be sequestered in special compartments such as vacuoles. Furthermore, increasing the antioxidant capacity of cells also helps the detoxification of heavy metals (Dietz et al., 1999).

The most effective way of detoxification involves specific transport proteins localized in the membranes. Recent studies have identified some genes encoding transport proteins from hyperaccumulators (Freeman et al., 2005; Mizuno et al., 2005) that are tested for their nickel transport activities in unicellular model organisms, like Escherichia coli and Saccharomyces cerevisiae. There are nickel transport proteins 
identified in maize, tobacco and Arabidopsis (Arazi et al., 1999; Forzani et al., 2001; von Wiren et al., 2006). These transporters have also been tested for their abilities to transport other metal ions such as $\mathrm{Zn}, \mathrm{Cd}$, $\mathrm{Mo}, \mathrm{Fe}, \mathrm{Pb}$, and have been shown to be capable of transporting some of these metals (Forzani et al., 2001; Mizuno et al., 2005; von Wiren et al., 2006).

In addition to the mechanisms mentioned above, secretory pathways, besides their involvement in crucial biological processes (protein folding, translocation, and endocytosis), are also shown to have important roles in heavy metal detoxification. The endoplasmic reticulum, Golgi, and small vesicles detoxify metals by removing them from the cytoplasm (Durr et al., 1998; Wu et al., 2002). For example, the Eca1 protein located in the endoplasmic reticulum leads to the uptake of manganese and confers manganese tolerance to the cells (Wu et al., 2002). Arabidopsis thaliana AtMTP11 (a member of Cation Diffusion Facilitator gene family) is another important example to highlight the importance of secretory pathway induced heavy metal detoxification system in plant cells (Peiter et al., 2007). AtMTP11 colocalizes with the Golgi-like compartments, and functions in removing manganese from cytosol into the endomembrane structures, and eventually pumps the element out of the cell via exocytosis (Peiter et al., 2007). Same researchers have also shown that Populus trichocarpa homolog of Arabidopsis Mtp11 protein, PtMtp11, function in the same manner (Peiter et al., 2007). Recently, a cDNA library study performed to identify Beta maritima manganese detoxification genes, added two more homologs to the MTP11 genes: BMMTP10 and BMMTP11. These two proteins have also been shown to localize and function in similar patterns to those Mtp11 proteins (Erbasol et al., 2013). Finally, in a very recently published study, researchers identified OsMTP8.1, which is the rice homolog of AtMTP11, and reported that OsMTP8.1 confers manganese tolerance via vacuole sequestering of the same element (Chen et al., 2013).

Recent studies on manganese tolerance clearly show the robustness of cDNA library based studies to identify heavy metal detoxification genes in plant species (Chen et al., 2013; Erbasol et al., 2013; Papoyan and Kochian, 2004; Pence et al., 2000). Apart from that, these studies have also shown the evolutionary importance and convergence of the secretory pathway proteins' significant involvement on the heavy metal detoxification (Chen et al., 2013; Erbasol et al., 2013; Peiter et al., 2007). Even though all these different plants do not share the same environment, and adapted to different levels of soil manganese levels, they all do have similar mechanism, and even share highly identical protein sequences to cope with heavy metal stress (Chen et al., 2013; Erbasol et al., 2013; Peiter et al., 2007).

Nickel, although is an essential element for plant growth, our knowledge on the nickel transport proteins is poor. With this motivation, we wanted to identify nickel homeostasis genes, in a plant (Beta vulgaris subspecies maritima) that has recently been shown to share conserved mechanisms to detoxify manganese (Erbasol et al., 2013), and is readily adapted to abiotic stress environments such as saline, manganese (Doney, 1993; Sillanpää, 1982). Thus we constructed a cDNA library of Beta maritima, and screened this library in the yeast system. We have identified NIC6 as a nickel tolerance gene, and a GFP fusion of Nic6 localized to multivesicular structures of tobacco cells and possibly acts in secretory pathway dependent nickel detoxification.

\section{Materials and methods}

\subsection{Yeast growth and media}

The yeast S. cerevisiae strain BY4741 (MATa his3 leu2 met15 ura3) was used in the experiments. Yeast cells were grown in either YPD media (with $2 \%$ glucose, $2 \%$ peptone, $1 \%$ yeast extract and $2 \%$ agar) or YNB (Yeast Nitrogen Base) minimal media including required amino acids and bases. For the solid media growth assays, wild type yeast cells were transformed with either empty vector pAG426GPD or NIC6
cDNA containing pAG426GPD over-expression vector. Yeast cells (shaken at $200 \mathrm{rpm}$, at $30{ }^{\circ} \mathrm{C}$ ), after overnight incubation, were diluted to $\mathrm{OD}_{600}=0.2,0.02,0.002,0.0002$ with sterile water, and five microliters of these cultures was transferred to spots on solid YNB (-ura; $2 \%$ glucose) plates without $\mathrm{NiCl}_{2}$ or with $\mathrm{NiCl}_{2}$ (with 5 different nickel concentrations between 500 and $1700 \mu \mathrm{M}$ ). Cells were incubated for five days at $30{ }^{\circ} \mathrm{C}$ and plates were photographed.

\subsection{Cloning and sequence analyses of NIC6 gene}

Cloning of the NIC6 cDNA was done using the Gateway Technology (Invitrogen, Karlsruhe, Germany). For expression purposes, the pAG426GPD (Addgene, USA) overexpression vector was used. Yeast transformation has been performed using the standard lithium acetate method (Kaiser et al., 1994). Blast searches were performed via the BLAST service of NCBI and for the multiple protein sequence alignments the T-coffee 6.85 (Notredame et al., 2000) software was used. The phylogenetic tree was calculated via MrBayes 3.1.2 (Huelsenbeck and Ronquist, 2001) and Newick format result of tree were given to TreeDyn 198.3 (Chevenet et al., 2006) for tree rendering. TMHMM Server v. 2.0 was used for prediction of membrane domains (Sonnhammer et al., 1998).

\subsection{Intracellular nickel measurements}

Intracellular nickel concentrations were measured as described by Mizuno (Mizuno et al., 2005). Pre-cultured yeast cells containing empty-vector or vector overexpressing NIC6 cDNA were grown overnight. Diluted solutions of cells were prepared at a ratio of $1 / 500$ in new YNB-ura medium that had been supplemented with $800 \mu \mathrm{M}$ $\mathrm{NiCl}_{2}$ and incubated for $30 \mathrm{~h}$. Then, cells were washed two times with $25 \mathrm{mM}$ EDTA and then were dried at $70{ }^{\circ} \mathrm{C}$ for $10 \mathrm{~h} .5$ milligrams of yeast cells was extracted using $\mathrm{HCl}$, filtered, 1:10 dilutions of filtrate were measured by ICP-MS, and result was given as $\mathrm{ppb}$.

\subsection{Plant growth, RNA isolation, and real-time PCR analyses}

B. maritima plants were grown in half-strength Hoagland Solution (Hoagland and Arnon, 1950) in a growth chamber under conditions of $12 \mathrm{~h}$ of dark and $12 \mathrm{~h}$ of light at $25{ }^{\circ} \mathrm{C}$. Earlier studies have shown that nickel treatment between $50 \mu \mathrm{M}$ and $200 \mu \mathrm{M}$ to diverse plant species (such as wheat, soybean, Brassica juncea, and Triticum aestivum) resulted in significant toxic effects on plant growth (for a detailed review, see Yusuf et al., 2011) those resulted in reduced shoot growth/mass, lowered shoot length, decreased dry mass, reduced germination rates, chlorosis and necrosis phenotypes etc. We have applied $75 \mu \mathrm{M} \mathrm{NiCl}_{2}$, which is not toxic level, was applied to the plants for seven days, leaf and root samples were collected every $24 \mathrm{~h}$. Total RNA isolation was performed using the Invitrogen RNA Isolation Kit (Invitrogen, Germany). DNase-treated total RNAs were used for cDNA synthesis using a cDNA Synthesis Kit (Fermentas, St. Leon-Rot, Germany). Maxima ${ }^{\mathrm{TM}}$ SYBR Green qPCR Master Mix was used (Fermentas, Germany) to perform the quantitative expression analyses of cDNAs using an IQ5 real-time PCR cycler system (Bio-rad, Germany). The Real-Time PCR primers used for amplification of NIC6 cDNA were NIC6RTF 5' AGCCTATCGTCC CACCAAAGTTGT-3' and NIC6RTR 5' ACATGGGTGTTCCCTTTCAGACCA-3' which are concurrently used to amplify 130 bp of cDNA. Real-time PCR primers for beta actin cDNA were: BmActRTF: 5'-AGACCTTCAATGTGCC TGCT-3' and BmActRTR 5'-TCAGTGAGATCACGACCAGC-3', and amplified 187 bp of B. maritima beta actin CDNA. The conditions for PCR amplification were as follows: $95{ }^{\circ} \mathrm{C} 5 \mathrm{~min}, 40$ cycles $94{ }^{\circ} \mathrm{C}$ for $25 \mathrm{~s}, 59^{\circ} \mathrm{C}$ for $25 \mathrm{~s}$, and $72{ }^{\circ} \mathrm{C}$ for $30 \mathrm{~s}$. 


\subsection{Construction of pENTR4 ${ }^{T M}$ vector}

The coding sequence for NIC6 was amplified by PCR from pAG426GPD using forward-primer 5'-AGACCATGGCTTCGATGGAGACAGAAAAGGG-3' and backward-primer 5'-AGACTCGAGTGGTATTTTGGGATGCGACGG-3'. The PCR primers used for amplification of Arabidopsis (AT4G15610) cDNA were AtNiGW6F 5' GGGGACAAGTTTGTACAAAAAAGCAGGCTATG GGTTATGAAACCAAATCGA-3' and AtNiGW6RGFP 5' GGGACCACTTTGTA CAAGAAAGCTGGGTCTCAACGGATTTTCTTGTAGAGG-3'.

Following a purification step the amplified gene fragment was digested with the restriction endonucleases NcoI and XhoI (restriction sites are underlined in primer sequences) and subsequently inserted in the corresponding restriction sites of pENTR4 ${ }^{\mathrm{TM}}$ GATEWAY cloning vector ${ }^{\circledR}$ (Invitrogen).

\subsection{Construction of pBatTL vector}

For the construction of the GATEWAY®-compatible binary pBatTL plant expression vector the Venus fragment was amplified by PCR using forward-primer 5'-AGAAGATCTATGGTTAGCAAAGGAGAAGAAC-3' and reverse-primer 5'-AGAAGATCTAAGATCCTCCTCAGAAATCAACTTTTGCTC TTTGTATAGTTCATCCATGCC-3' for N-terminal Venus (restriction sites are underlined and the myc sequences are shown in italics). The corresponding fragment was then inserted into the BglII site of the Gateway (GW) vector for N-terminal fusions to obtain pBatTL-Venus-(GW). The NIC6 construct was introduced into the pBatTL vector by recombination and verified by sequencing (Sanger, 1977) using an ABI PRISM® 3100 Genetic Analyzer (Applied Biosystems).

\subsection{Transient expression in plants}

Transient expression of the fluorescence construct was carried out in intact plant leaves of 4-week-old Nicotania benthamiana by co-infiltration with Agrobacterium tumefaciens strain GV3101 containing the Venus-NIC6 construct in combination with the C58C1 strain carrying the pCH32 helper plasmid which contains the RNA silencing suppressor protein p19 of tomato bushy stunt virus (Voinnet et al., 2003). Fluorescence was observed 3 days after infiltration by confocal laser scanning microscopy (CLSM) with a Leica DMIRE2 microscope (Leica Microsystems) using the excitation/ emission wavelengths of 514/540-580 nm.

\subsection{GFP fusion protein localization in yeast}

Full-length coding sequences for NIC6 were amplified using the following primers, NiGW6F 5'-GGGGACAAGTTTGTACAAAAAAGCAGGCTA TGTCATCGATGGAGACAGAAAAGG-3' and NiGW6RGFP-5'-GGGGACCA CTTTGTACAAGAAAGCTGGGTCGTATTTTG GGATGCGACGGTAAAG-3' that contained the Gateway-compatible attachment sites. Following the Gateway protocol (Invitrogen), this PCR fragment was cloned into destination vector PAG413GPD-ccdB-EGFP (Addgene). The resulting plasmid was transformed into yeast. Fluorescence was observed 3 days after infiltration by confocal laser scanning microscopy (CLSM) with a Leica DMIRE2 microscope (Leica Microsystems).

\section{Results}

\subsection{Identification of NIC6 as a nickel tolerance gene}

We screened cDNA library of B. maritima to identify plant genes that confer nickel tolerance to yeast. Transformed yeast cells were plated onto selective media containing $1500 \mu \mathrm{M}$ of nickel, which is a toxic level for wild type cells, and only three colonies were able to grow. After streaking these colonies out onto new nickel plates we have decided to continue with one candidate which confirmed growth phenotype on toxic level of nickel media. Plasmids were recovered from these transformed cells, sequenced with vector-based primers and the cDNA, named as NIC6 (ADA71986), was recovered. Spotting assay results confirmed that NIC6 overexpressing cells were able to grow in the presence of $1700 \mu \mathrm{M}$ nickel (Fig. 1). The NIC6 open reading frame consists of 582 base pairs and encodes for a polypeptide, which consists of 194 amino acids. Domain analyses of this polypeptide suggested Nic6 as a membrane protein with four trans membrane domains. The Blastp result of Nic6 showed homology with a putative integral membrane protein. Alignment result and phylogenetic relations within these 6 homologous proteins are given in Figs. 2A and B. Out of these six homolog proteins, only Ab267825 (common name InPsr26p) has a known function, which plays role in programmed cell death in the Ipomoea nil plant (Shibuya et al., 2009). We also searched for similar proteins in A. thaliana to see if there are any homolog proteins with known functions. All of the identical proteins are likely to be integral membrane proteins but the functions of these proteins are unknown. One Arabidopsis homolog (AT4G15610) is also overexpressed in yeast cells to see whether this homolog also has a nickel detoxification role, but AT4G15610 overexpressing yeast cells did not show any nickel tolerant phenotype (Data not shown).

\subsection{Characterization of Nic6 activity}

Genes playing role in heavy metal stress tolerance often provide cross resistance to many metal ions. In order to test whether Nic6 plays role in tolerance to other metals, we treated the transformants with toxic levels of cobalt, cadmium, and zinc. As seen in Figs. 3A, B, and C, NIC6 overexpressing cells were not resistant to cobalt, cadmi$\mathrm{um}$, and zinc treatments. We also measured intracellular nickel concentration of yeast cells (either containing an empty-vector or NIC6 overexpressing vector) treated with nickel to see if it functions as an efflux pump protein since it showed possible membrane localization. Statistical analysis (Two-tailed $t$-test, $\mathrm{df}=6, \mathrm{P}=0.3027$ ) showed no significant differences between the control and NIC6 overexpressing cells (Fig. 4), and the reason for this result was further proven later on under cellular localization of Nic6 protein in yeast and tobacco cells.

\subsection{Expression analysis of NIC6 in B. maritima}

Next, we wanted to know whether NIC6 expression is regulated via nickel in plant cells. Real Time PCR analyses of NIC6 mRNA was performed on leaves and roots of $B$. maritima grown in half strength Hoagland solution (Hoagland and Arnon, 1950) containing $75 \mu \mathrm{M}$ nickel for 3 days. Addition of $75 \mu \mathrm{M}$ nickel to the plant growth medium did not alter NIC6 transcript levels neither in leaf nor in root tissue (Figs. 5A and B). This suggests that NIC6 regulation was not directly induced via nickel signalling.

\subsection{Cellular localization of Nic6 protein in tobacco and yeast cells}

Since Nic6 is a possible putative integral membrane protein, finally, we investigated the localization of the protein in plant and yeast cells. We monitored sub-cellular localization of Nic6 by expressing a Venus-Nic6 fusion protein construct in Nicotiana benthamiana epidermal cells. The entire coding region of NIC6 was translationally fused to the carboxy-terminus of the fluorescent Venus protein and placed under the control of the double enhanced CaMV $35 S$ promoter. Subsequently, the resulting construct was transformed into $N$. benthamiana leaves by agro infiltration in the presence of the potent RNA gene silencing suppressor protein 19 from tomato bushy stunt virus and confocal laser scanning microscopy was used to visualize the fluorescence signals. Fig. 6 representatively shows the outcome of three individual experiments. Fluorescence mediated by the Nic6-Venus fusion protein was specifically localized to the surface of multivesiculare vesicles (Figs. 6A-E). These structures are clearly localized within the cell 


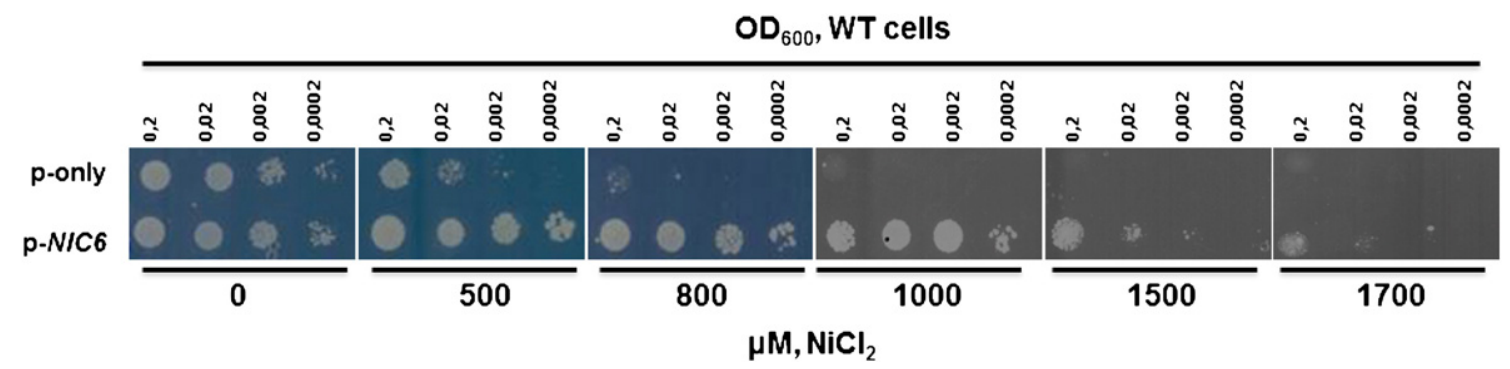

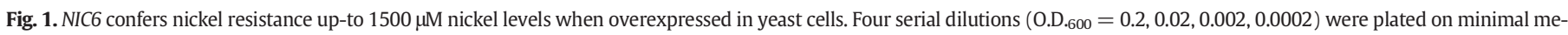
dium. Cells were incubated for 5 days at $30^{\circ} \mathrm{C}$.

cytoplasm as demonstrated by the co-expression of CaMV35S-mRFP constructs, which led to an overall red fluorescence of the cytoplasm (Figs. 6F-I). To distinguish the multivesiculare vesicles from chloroplasts, the auto-fluorescence of the chlorophyll is shown as blue fluorescence signal (Fig. 6J).

We have fused GFP protein to the NIC6 cDNA, and transformed NIC6-GFP construct into the BY4741 yeast strain to monitor the yeast cell localization analysis of the protein. Fig. 6L shows the yeast cellular localization of the Nic6-GFP fusion protein. Construct accumulates as a punctuated pattern in the cytosol. As similar to the tobacco epidermal cell localization results, the yeast localization also might point to the endomembrane structures derived from secretory pathway members such as Golgi or endoplasmic reticulum.

\section{Discussion}

In this study, we screened a cDNA library of $B$. maritima to identify plant genes that confer nickel tolerance in yeast $S$. cerevisiae cells, and Nic6 was identified as a nickel tolerance protein. The only homologous protein (Fig. 2) with a known function is the InPSR26 of the Japanese morning glory (Ipomoea nil), showed 53\% sequence identity at the amino acid level (Shibuya et al., 2009). The InPSR26 has a regulatory role in programmed cell death and autophagy (Shibuya et al., 2009), and we currently do not know whether NIC6 gene product has any roles in autophagy pathway in plants.

The most effective way of detoxification involves specific transport proteins localized in the membranes. Recent studies have identified some genes encoding transport proteins from hyperaccumulators (Freeman et al., 2005; Mizuno et al., 2005) those are tested for their nickel transport activities in unicellular model organisms, like E. coli and $S$. cerevisiae. There are also nickel transport proteins identified in maize, tobacco and Arabidopsis (Arazi and Fromm, 1999; Forzani et al., 2001; von Wiren et al., 2006). These transporters have also been tested for their abilities to transport other metal ions such as $\mathrm{Zn}, \mathrm{Cd}$, Mo, Fe, or $\mathrm{Pb}$ and have been shown to be capable of transporting some of these metals (Forzani et al., 2001; Mizuno et al., 2005; von Wiren et al., 2006). Since NIC6 encodes for a potential membrane protein, we reasoned that it might be playing role as an efflux pump, transports nickel

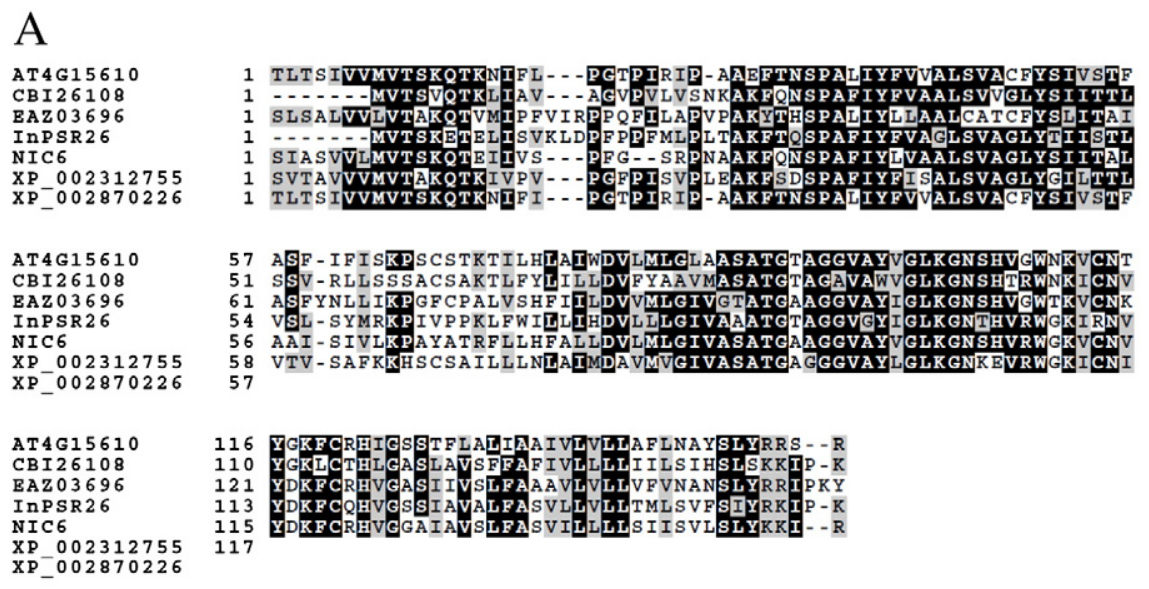

B

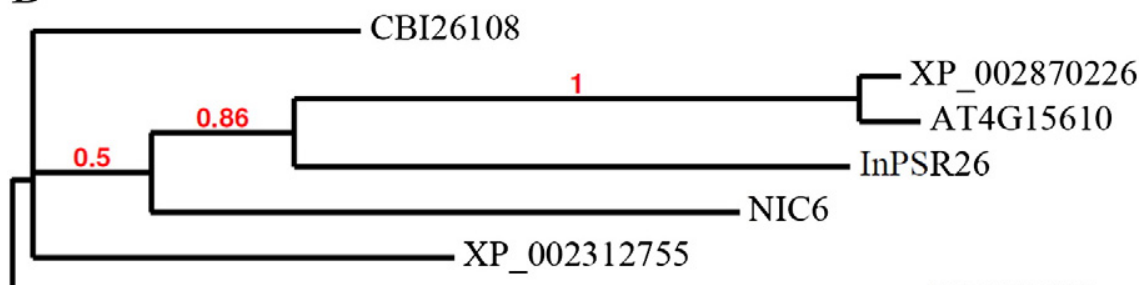

EAZ03696

\section{3}

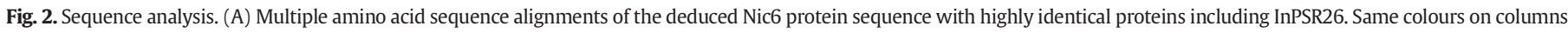

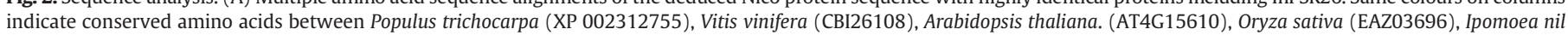
(InPSR26), Arabidopsis lyrata (XP 002870226) and Beta maritima (Nic6). (B) Phylogenetic tree of the Nic6 and homolog proteins. 
A

$\mathrm{OD}_{600} \Delta$ cot1 cells

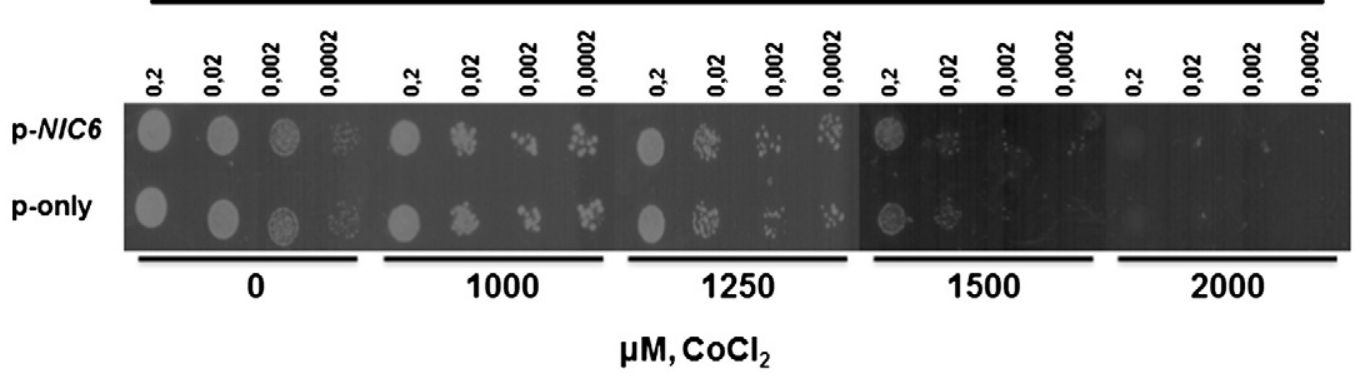

B

$\mathrm{OD}_{600}, \Delta y c f 1$ cells

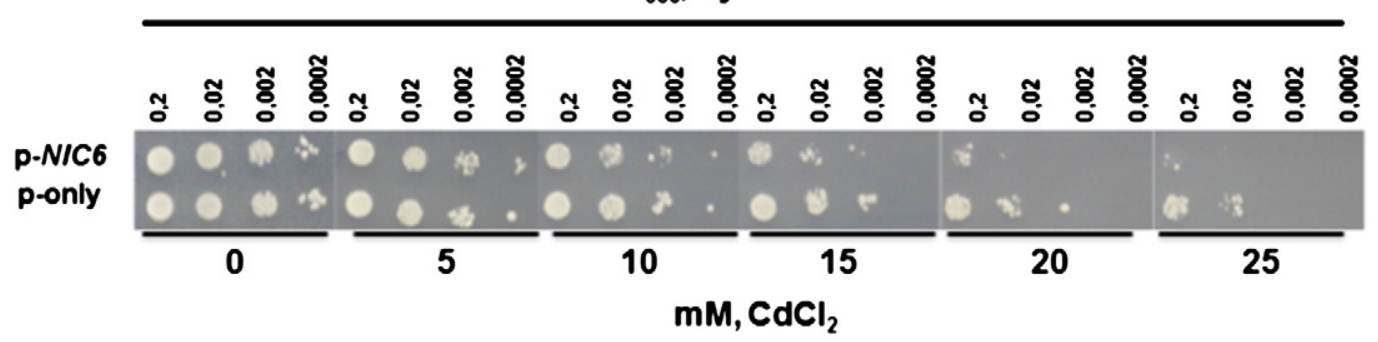

$\mathrm{C}$

$\mathrm{OD}_{600} \Delta \mathrm{zhy3}$ cells

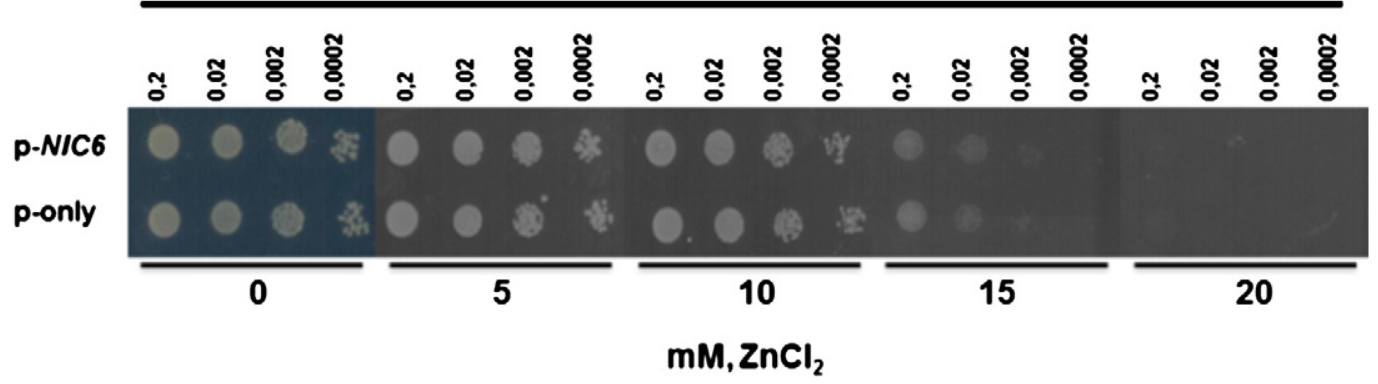

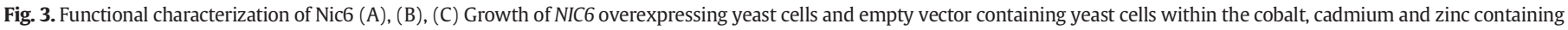
YNB-ura plates, respectively (isogenic $\Delta \cot 1, \Delta y c f 1$, and $\Delta$ zhy3 mutant cells were used for cobalt, cadmium, and zinc stress experiments).

from cytoplasm to outside, and decreases intracellular nickel levels. However, nickel levels of both sham vector containing and NIC6

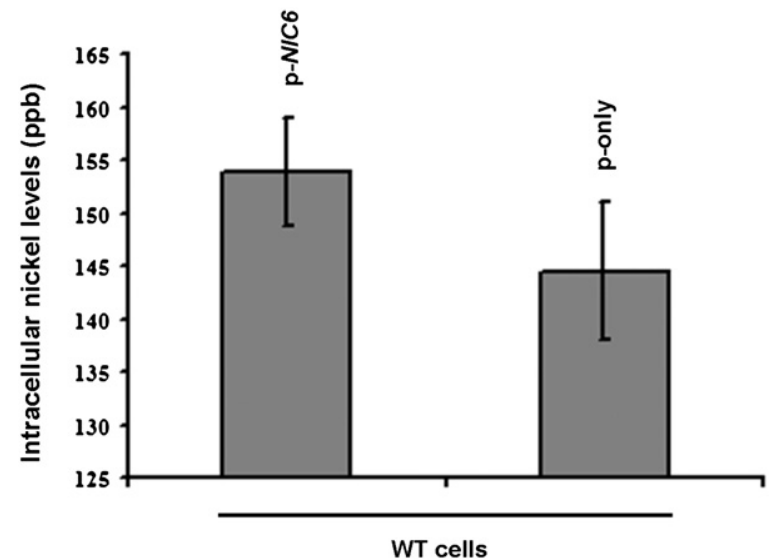

Fig. 4. Intracellular nickel concentrations of recombinant yeast cells showing that there is no significant difference between control and NIC6 overexpressing cells within 95\% confidence level (Two-tailed $t$-test, $\mathrm{df}=6, \mathrm{P}=0.3027$ ). Control cells contain empty pAG426GPD vector and NIC6 cells are overexpressing NIC6 cDNA. Values are the mean of two independent experiments as 2 replicates each $(\mathrm{SEM} \pm, \mathrm{n}=4)$. overexpressing cells (Fig. 4) were not statistically different (Two-tailed $t$-test, $\mathrm{df}=6, \mathrm{P}=0.3027$ ). Delhaize and colleagues (Delhaize et al., 2007) also observed a similar result: intracellular $\mathrm{Mn}^{2+}$ concentration stayed at similar levels between yeast cells expressing AtMTP11 and yeast cells with empty vector. This supports our results as, Nic6 does not export nickel to the outside of the cell but it enhances nickel tolerance via different strategies such as internal sequestration of heavy metals in the cell.

Detoxification proteins usually play role in detoxification of more than one substrate. In order to test if Nic6 function is specific for only nickel, NIC6 overexpressing yeast cells were grown in cobalt, cadmium, and zinc media, but Nic6 conferred tolerance only against nickel toxicity (Fig. 3). Different metals need to be tested other than cobalt, cadmium, and zinc. We have also analysed Arabidopsis homolog of Nic6, At4g15610, to see nickel tolerance and found that Arabidopsis protein did not confer nickel tolerance in yeast. This is similar to the Stylosanthes hamata metal tolerance protein ShMtp1's case. This protein is a member of CDF transporter family, and detoxifies manganese in this plant (Delhaize et al., 2003). ShMtp1 shares high sequence similarity with Arabidopsis and rice CDF proteins, but only ShMtp1 protein has manganese detoxification activity which is parallel to our results with NIC6. Some of the metal transporter genes are transcriptionally activated upon treatment with its 


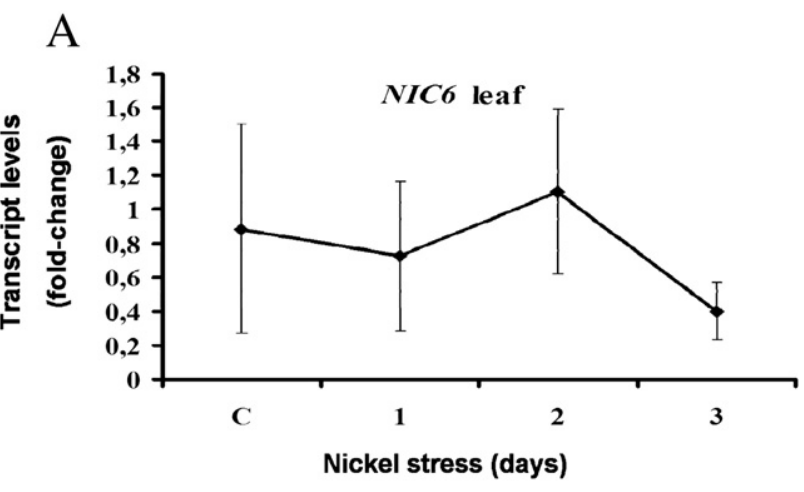

B

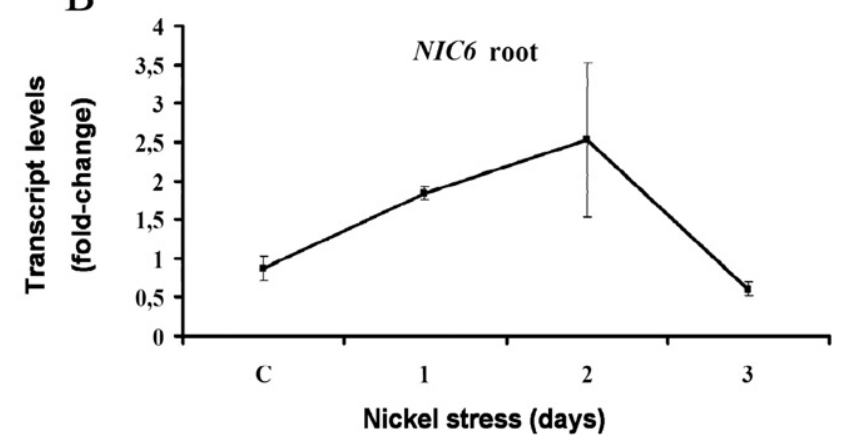

Fig. 5. Expression levels of NIC6 mRNA after $75 \mu \mathrm{M}$ nickel treatment of Beta maritima. (A) Expression of the NIC6 CDNA in the leave from control (without Ni) to 3 days nickel treatment in the leave. (B) Expression of the Nic6 cDNA in the root. Error bars represent the standard error of the mean $(n=4)$. stressor. AtHMA4, a Zn-transporting ATPase, is up-regulated when Zn levels increased (Williams and Mills, 2005), and IRT1, Fe transporter, is up-regulated under low Fe conditions (Vert et al., 2001). Whereas, MTP1/ZAT transcript levels were not altered by metals (van der Zaal et al., 1999), and transcript levels of AtMTP11, cation diffusion facilitator (CDF) family protein which transports manganese, were not increased in COL 0 wild-type Arabidopsis plant under toxic manganese stress (Sanders et al., 2007). Our expression analysis also showed similar kind of expression patterns. In leaves (Fig. 5A), NIC6 transcript levels were not induced via nickel stress within three days compared to the untreated control. But in root cells, more dynamic expression was seen due to the nickel treatment (Fig. 5B). But even though a more dynamic regulatory pattern was observed in the root cells compared to the leaf cells, expression analysis is not enough to prove a direct induction of NIC6 transcription via nickel stress. It would have been more informative to perform the expression analysis in separate for young and old tissues. Although it is specifically related to the plant species (Yusuf et al., 2011), there has been some research showing that, nickel as being a highly mobile element, might be immediately transported to newly formed tissues (Riesen and Feller, 2005) Thus, nickel induced induction of NIC6 transcripts, if there is, might have been more transparent in the young root and leaf tissues, compared to the older tissues. But still, using the control samples, which were also, as nickel treated samples, collected both from the young and old tissues rule out this masking effect.

Finally to gain a better understanding of the detailed localization of the Nic6 protein, we cloned cDNA to the upstream regions of GFPand YFP-fusion proteins, and then transformed these constructs to the tobacco epidermal cells. From the results (Figs. 6A-J), it can be concluded that the Nic6 protein in plant and yeast localizes in multivesicular
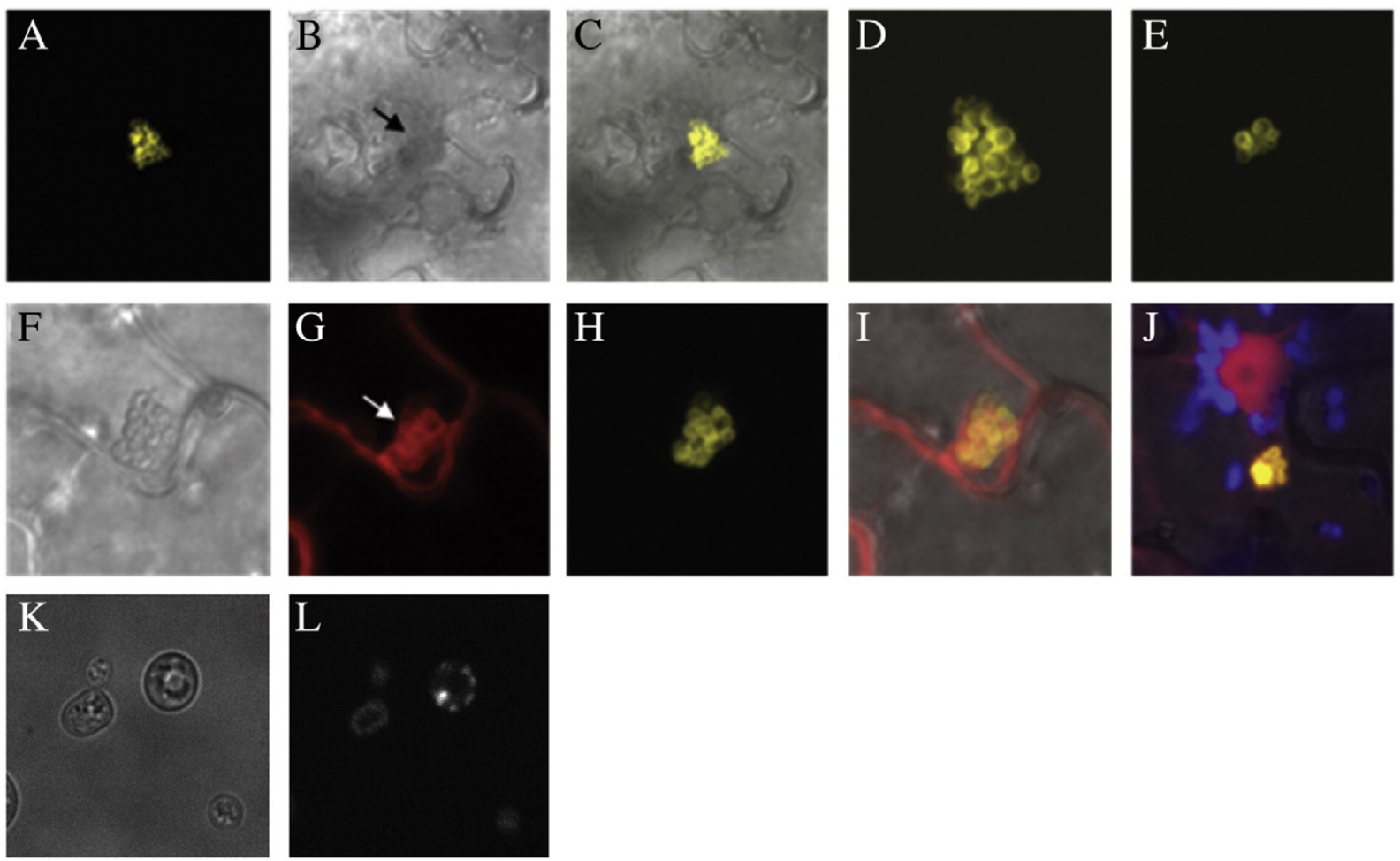

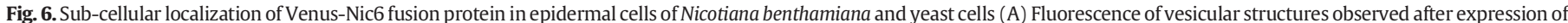

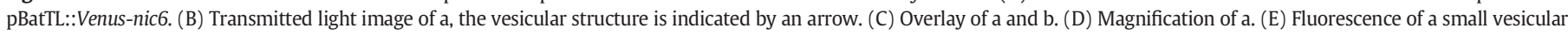

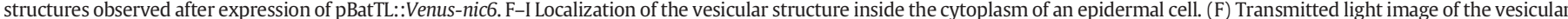

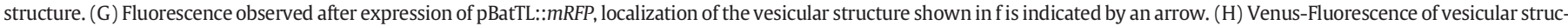

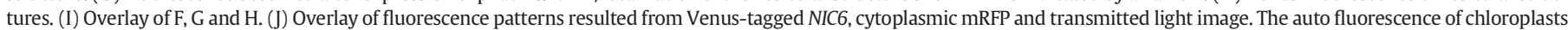
is shown in blue. (K) Transmitted light image of yeast cells. (L) Sub-cellular localization of Nic6-GFP fusion protein in yeast cells. 
protein bodies, which may derive from endoplasmic reticulum, tonoplast, peroxisomes, or liposomes. Although there are limited findings in the literature, it is known that coated vesicles, endomembrane structures have been shown to transport metal ions such as $\mathrm{Ca}^{2+}$ and $\mathrm{Mn}^{2+}(\mathrm{Wu}$ et al., 2002) and confer $\mathrm{Mn}^{2+}$ tolerance. During the transport of metals through these endomembrane structures, first, cells remove these toxic elements from cytoplasm where active metabolism takes place. And in some cases, with the transfer of these endomembrane systems through the plasma membrane, even the contents of these structures are removed from the cell itself. Arabidopsis homolog of Nic6, At4g15610, showed homology with Casparian strips (CASP) genes (Roppolo et al., 2011). CASP5GFP localized large, irregular intracellular compartments in the cell. The authors speculated that CASP form polymeric scaffold within the membrane and serve as a platform to localize and immobilize cell wall biosynthetic enzymes and interacting proteins. Localization of Nic6 showed multivesicular structures closer to the plasma membrane similar to CASP5 protein localization. Nic6 might be serves as CASP like function and help nutrient and nickel uptake into root cells.

All in all, our analyses show that Nic6 confers nickel resistance to yeast cells, and localizes to the multivesicular bodies of tobacco epidermal cells and were not significantly induced via nickel treatment in B. maritima cells. Detailed detoxification mechanisms by which Nic6 operates need to be further investigated.

\section{Conflict of interest}

\section{All authors declare no conflict of interest.}

\section{Acknowledgements}

We thank Dr. Serdar Özçelik (Izmir Institute of Technology, Izmir, Turkey) for the help with confocal fluorescence microscopy and nickel measurements, the biotechnology core facility and Biological Mass Spectrophotometry facility of the Izmir Institute of Technology for the help with instruments, and Dr. Joachim Uhrig and Dr. Guido Jach (Cologne) for providing the pBatTL plant expression vector. We thank to Çiğdem Işık for the helps on cadmium spotting assay. Finally, we thank to Dr. Ritchie Eanes for the critical reading of the manuscript.

This work was supported by IYTE-BAP and DPT (T.R. Prime Ministry State Planning Organization).

\section{References}

Arazi, T., Fromm, H., 1999. Characterization of a novel family of calmodulin-binding plasma membrane channel-like proteins. Plant Biotechnology and in Vitro Biology in the 21st Century, 36 351-353.

Arazi, T., Sunkar, R., Kaplan, B., Fromm, H., 1999. A tobacco plasma membrane calmodulin-binding transporter confers $\mathrm{Ni} 2+$ tolerance and $\mathrm{Pb} 2+$ hypersensitivity in transgenic plants. Plant J. 20, 171-182.

Boominathan, R., Doran, P.M., 2002. Ni-induced oxidative stress in roots of the $\mathrm{Ni}$ hyperaccumulator, Alyssum bertolonii. New Phytol. 156, 205-215.

Chen, Z., et al., 2013. Mn tolerance in rice is mediated by MTP8.1, a member of the cation diffusion facilitator family. J. Exp. Bot 64, 4375-4387.

Chevenet, F., Brun, C., Banuls, A.L., Jacq, B., Christen, R., 2006. TreeDyn: towards dynamic graphics and annotations for analyses of trees. BMC Bioinform. 7.

Delhaize, E., Kataoka, T., Hebb, D.M., White, R.G., Ryan, P.R., 2003. Genes encoding proteins of the cation diffusion facilitator family that confer manganese tolerance. Plant Cell 15, $1131-1142$.

Delhaize, E., et al., 2007. A role for the AtMTP11 gene of Arabidopsis in manganese transport and tolerance. Plant J. 51, 198-210.

Dietz, K.J, Baier, M., Krämer, U., 1999. Free radicals and reactive oxygen species as mediators of heavy metal toxicity in plants. In: Prasad, M.N.V., Hagemeyer, J. (Eds.), Heavy metal stress in plants: from molecules to ecosystems. Springer, Verlag, Berlin, pp. 73-97.
Dixon, N.E., Gazzola, C., Blakeley, R.L., Zerner, B., 1975. Jack-Bean urease (Ec 3.5.1.5) metalloenzyme - simple biological role for nickel. J. Am. Chem. Soc. 97, 4131-4133. Doney, D.L., 1993. Broadening the genetic base of sugarbeet. J. Sugarbeet Research 30 , 209-219.

Durr, G., et al., 1998. The medial-Golgi ion pump Pmr1 supplies the yeast secretory pathway with $\mathrm{Ca} 2+$ and $\mathrm{Mn} 2+$ required for glycosylation, sorting, and endoplasmic reticulum associated protein degradation. Mol. Biol. Cell 9, 1149-1162.

Erbasol, I., Bozdag G.O, Koc, A., Pedas, P. Karakaya, H.C, 2013. Characterization of two genes encoding metal tolerance proteins from Beta vulgaris subspecies maritima that confers manganese tolerance in yeast. Biometals 26, 795-804.

Eskew, D.L., Welch, R.M., Norvell, W.A., 1984. Nickel in higher-plants - further evidence for an essential role. Plant Physiol. 76, 691-693.

Forzani, C., Loulergue, C., Lobreaux, S., Briat, J.F., Lebrun, M., 2001. Nickel resistance and chromatin condensation in Saccharomyces cerevisiae expressing a maize high mobility group I/Y protein. J. Biol. Chem. 276, 16731-16738.

Freeman, J.L., Persans, M.W., Nieman, K., Salt, D.E., 2005. Nickel and cobalt resistance engineered in Escherichia coli by overexpression of serine acetyltransferase from the nickel hyperaccumulator plant Thlaspi goesingense. Appl. Environ. Microbiol. 71, 8627-8633.

Hoagland, D.R., Arnon, D.I., 1950. The Water-culture Method for Growing Plants without Soil. Circ., Univ. of Calif. Agric. Exp. Station, Berkley 437.

Huelsenbeck, J.P., Ronquist, F., 2001. MRBAYES: Bayesian inference of phylogenetic trees. Bioinformatics 17, 754-755.

Kaiser, C., Michaelis, S., Mitchell, A., 1994. Methods in Yeast Genetics. Cold Spring Harbor Laboratory Press, New York.

Mizuno, T., Usui, K., Horie, K., Nosaka, S., Mizuno, N., Obata, H., 2005. Cloning of three ZIP/ NRAMP transporter genes from a Ni hyperaccumulator plant Thlaspi japonicum and their Ni2 + - transport abilities. Plant Physiol. Biochem. 43, 793-801.

Notredame, C., Higgins, D.G., Heringa, J., 2000. T-Coffee: a novel method for fast and accurate multiple sequence alignment. J. Mol. Biol. 302, 205-217.

Papoyan, A., Kochian, L.V., 2004. Identification of Thlaspi caerulescens genes that may be involved in heavy metal hyperaccumulation and tolerance. Characterization of a novel heavy metal transporting ATPase. Plant Physiol. 136, 3814-3823.

Peiter, E., et al., 2007. A secretory pathway-localized cation diffusion facilitator confers plant manganese tolerance. Proc. Natl. Acad. Sci. U. S. A. 104, 8532-8537.

Pence, N.S., et al., 2000. The molecular physiology of heavy metal transport in the $\mathrm{Zn} / \mathrm{Cd}$ hyperaccumulator Thlaspi caerulescens. Proc. Natl. Acad. Sci. U. S. A. 97, 4956-4960.

Peralta-Videa, J.R., Gardea-Torresdey, J.L., Gomez, E., Tiemann, K.J., Parsons, J.G., Carrillo, G., 2002. Effect of mixed cadmium, copper, nickel and zinc at different pHs upon alfalfa growth and heavy metal uptake. Environ. Pollut. 119, 291-301.

Riesen, O., Feller, U., 2005. Redistribution of nickel, cobalt, manganese, zinc, and cadmium via the phloem in young and maturing wheat. J. Plant Nutr. 28, 421-430.

Roppolo, D., et al., 2011. A novel protein family mediates Casparian strip formation in the endodermis. Nature 473, 380-U564.

Sajwan, K.S., Ornes, W.H., Youngblood, T.V., Alva, A.K., 1996. Uptake of soil applied cadmium, nickel and selenium by bush beans. Water Air Soil Pollut. 91, 209-217.

Sanders, D., et al., 2007. A secretory pathway-localized cation diffusion facilitator confers plant manganese tolerance. Proc. Natl. Acad. Sci. U. S. A. 104, 8532-8537.

Sanger, F., 1977. Nucleotide-sequence of DNA of Ox174. Fed. Proc. 36, 266

Seregin, I.V., Kozhevnikova, A.D., 2006. Physiological role of nickel and its toxic effects on higher plants. Russ. J. Plant Physiol. 53, 257-277.

Shibuya, K., Yamada, T., Suzuki, T., Shimizu, K., Ichimura, K., 2009. InPSR26, a putative membrane protein, regulates programmed cell death during petal senescence in Japanese morning glory. Plant Physiol. 149, 816-824.

Sillanpää, M., 1982. Micronutrients and the nutrient status of soils: a global study. Food and Agriculture Org. Rome (48).

Sonnhammer, E.L.L., Eddy, S.R., Birney, E., Bateman, A., Durbin, R., 1998. Pfam: multiple sequence alignments and HMM-profiles of protein domains. Nucleic Acids Res. 26, 320-322.

van der Zaal, B.J., et al., 1999. Overexpression of a novel Arabidopsis gene related to putative zinc-transporter genes from animals can lead to enhanced zinc resistance and accumulation. Plant Physiol. 119, 1047-1055.

Vert, G., Briat, J.F., Curie, C., 2001. Arabidopsis IRT2 gene encodes a root-periphery iron transporter. Plant J. 26, 181-189.

Voinnet, O., Rivas, S., Mestre, P., Baulcombe, D., 2003. An enhanced transient expression system in plants based on suppression of gene silencing by the $\mathrm{p} 19$ protein of tomato bushy stunt virus. Plant J. 33, 949-956.

von Wiren, N., Schaaf, G., Honsbein, A., Meda, A.R., Kirchner, S., Wipf, D., 2006. AtIREG2 encodes a tonoplast transport protein involved in iron-dependent nickel detoxification in Arabidopsis thaliana roots. J. Biol. Chem. 281, 25532-25540.

Walsh, C.T., Ormejohnson, W.H., 1987. Nickel enzymes. Biochemistry 26, 4901-4906.

Watt, R.K., Ludden, P.W., 1999. Nickel-binding proteins. Cell. Mol. Life Sci. 56, 604-625.

Williams, L.E., Mills, R.F., 2005. P-1B-ATPases - an ancient family of transition metal pumps with diverse functions in plants. Trends Plant Sci. 10, 491-502.

$\mathrm{Wu}$, Z.Y., et al., 2002. An endoplasmic reticulum-bound Ca2 +/Mn2 + pump, ECA1, supports plant growth and confers tolerance to Mn2 + stress. Plant Physiol. 130, 128-137.

Yusuf, M., Fariduddin, Q., Hayat, S., Ahmad, A., 2011. Nickel: an overview of uptake, essentiality and toxicity in plants. Bull. Environ. Contam. Toxicol. 86, 1-17. 